paralysis of 20 myodioptres. The curve of homatropin paralysis has a very steep ascent and a slow descent while in cocain both ascent and descent are steep (Fig. 5).

The curves illustrate many other interesting points such as the influence of heterophoria, the stage of paralysis at which the uniocular and binocular near-points differ, and the difficulty in reading experienced by persons who have been ill or who are hypermetropic, although they appear to have sufficient accommodative power.

We can hardly hope that such measurements, the technical difficulty of which is so considerable, can ever be of much clinical use but we hope to have shown that the careful study of relative accommodation by such methods can give us a more accurate view on several questions concerning accommodation.

\title{
THE COMPARATIVE VALUE OF COCAIN SUBSTITUTES*
}

\author{
BY \\ R. Foster MoOre \\ London
}

I wiLl endeavour to be as brief as possible in what I have to say, for it seems to me that this discussion will best serve its purpose, if the experience of everyone here, to the exclusion of theoretical considerations, can be made available to our knowledge; the combined experience encompassed by this meeting must represent many thousands or perhaps hundreds of thousands of observations.

I judge from the published title that it is cocain substitutes and not cocain itself which are to form the subject of discussion. I imagine, however, that we shall all feel disposed to use cocain as our standard of reference, and to speak of substitutes in terms of this adopted standard.

The following are the most important drugs which have been used for local anaesthesia in addition to cocain :

(1) Holocain.

(2) $\alpha$ and $\beta$ Eucain.

(3) Euphthalmin.

(4) Novocain.

(5) An'aesthesin.

(6) Subcutin.

(7) Antinesin.

${ }^{*}$ Contribution to a Discussion held at the Royal Society of Medicine on December 7, 1923. 
(8) Alypin.

(9) Stovain.

(10) Tropacocain.

(11) Butyn.

I have details here with respect to all of these drugs as regards their reputed anaesthetic powers, toxicity, liability to become drugs of addiction, action on the corneal epithelium, etc., etc. It is clearly impossible to give them now, and I do not think it would assist us in our discussion.

Local anaesthetics are used in different ways and in different strengths by the members of the various sections represented, but there are one or two points which are common ground about which valuable information may be forthcoming.

First, I would suggest the important question of addiction, as to whether anyone present has certain knowledge of cases where addiction has resulted from his own use of cocain for surgical purposes, or as a result of his prescribing it, or whether he has knowledge of any case of addiction in respect of any of the cocain substitutes.

This latter is a point of considerable importance, for these drugs are little known, and were cocain not available, it might well be that one or other of them would be found to have an effect which appealed to the narcomaniac. It may, moreover, be said that there is at present some degree of inducement to use them in this way, for there are not the penalties attached to the possession of them that hold in the case of cocain, and hence, if it can be show $n$ that in spite of their accessibility there is no abuse of them, it would be evidence of some weight that they are not addictable drugs.

A second point of common ground is the degree of toxicity of the various drugs.

Other important points for our guidance have been put forward by $\mathrm{Mr}$. Watson-Williams.

From the ophthalmic point of view we have to consider whether there is a drug, or combination of drugs, which, when weighed against cocain, is its equal or superior in respect of the following points :

(a) Degree of anaesthesia, and the rapidity with which this is attained.

(b) The amount of irritation caused, remembering that some of the drug may get inside the eye during an operation and cause iritis afterwards.

(c) Whether it is more or less liable to cause desquamation of the corneal epithelium.

(d) Whether it is as well absorbed if the eye is not quite free from injection. 
(e) Toxicity.

(f) Stability, whether it can be sterilized by boiling.

$(g)$ Whether it stimulates the sympathetic nerve.

(h) Its liability to become a drug of addiction.

May I state briefly the chief uses of local anaesthetics in ophthalmic work.

(1) Instillation anaesthesia.-This is the most important method of use, solutions of various strengths being dropped into the conjunctival sac. Anaesthesia produced in this way is made use of for tonometric examinations, for removal of foreign bodies from the conjunctival sac or cornea, for cauterization of the cornea, for syringing the lacrymal ducts, and, by a long way the most important, for a group which we may include under the head of "all intraocular operations on uninflamed eyes, except where the patient is so young as to render general anaesthesia desirable."

It is this last group of intraocular operations which I believe is the crux of the whole question for the ophthalmic surgeon, for I am sure there are a number of cocain substitutes which are sufficiently efficacious for surface anaesthesia.

(2) Infiltration anaesthesia.-This method is used in plastic operations on the lids, for meibomian cysts, in a few cases for intraocular operations on inflamed eyes and enucleation of the eyeball where a general anaesthetic for some reason is not available, and for injecting into the orbicularis as a preliminary to a cataract extraction to produce temporary paralysis of the muscle and so prevent squeezing on the part of the patient.

It seems to me for all, these purposes novocain in 1 per cent. or 2 per cent. solution, is on the one hand so efficient, and on the other so little toxic, that cocain does not enter into competition with it for this part of ophthalmic work, and I would suggest that time will be saved if it be eliminated from the discussion, we have, however, to consider whether it may take the place of cocain when used by instillation.

(3) Used as a solid.-Cocain hydrochloride is used in its solid form for topical application in incising meibomian cysts and in muscle operations; the point to consider here is to what extent the solid form of other drugs can be used in the same way, or whether novocain injections are not equally satisfactory for these purposes.

Another quality of cocain is its power of stimulating the sympathetic nerve, producing dilatation of the pupil and widening of the palpebral fissure. It is sometimes claimed as an advantage of other drugs that they have no action on the pupil, but I fancy most observers will consider both this action and the 
manner in which cocain can be used as a test for paralysis of the sympathetic nerve, as a valuable quality. Information with regard to other drugs in this respect would be valuable.

It has been suggested to me that the stimulating action on the sympathetic nerve might prove to be a test of the power of a drug to act as a stimulant of the central nervous system, and that so it might become a test of the liability of a drug to become a drug of addiction, a valuable test should it be substantiated.

My own experience of cocain substitutes is as follows :-

I have already expressed my opinion of novocain for infiltration ; but have not used it for instillation. I have found holocain in 2 per cent. solution satisfactory for superficial anaesthesia by instillation, but it causes distinctly more smarting than cocain of similar strength; I have not used it for intraocular operations. I have used 2 per cent. and 4 per cent. stovain by instillation both for superficial anaesthesia and for cataract operations, it is more irritating than cocain, it gives a satisfactory superficial anaesthesia, but I have thought it unsatisfactory for cataract operations, and on two occasions have had to complete the operation with the aid of cocain. I have used butyn a few times in the form of gelatin lamellae and 2 per cent. drops for superficial anaesthesia only, the anaesthesia seemed satisfactory but smarting was rather marked.

I know of no certain case of addiction to any of these drugs as a result of their clinical use by myself or others, the most I can say of one case is that I suspect the cocain habit was contracted as a result of the use of cocain ointment for a fistula-in-ano.

\title{
THE RADIOGRAPHIC LOCALIZATION OF FOREIGN BODIES IN THE EYE
}

\author{
BY \\ Professor Dr. L. DE LiebermaNi \\ BUDAPEST
}

In a previous paper dealing with the localization of foreign bodies in the eye (Arch. $f$. Augenheilk., Vol. LXXVI, p. 1ri) I gave a detailed description of the Sweet method, a method then little known in Europe though, in my opinion, one of the best. Sinice then it has become more popular. In this paper I emphasized the importance of making the exposure as brief as possible in order to prevent any movement from the exact optical direction. The longer the exposure the greater the risk of the patient losin? the fixation point by movement of the head or eye. I next 